\title{
Conductas parentales y habilidades sociales en estudiantes de educación secundaria del Callao
}

\section{Parental Behaviors and Social Skills in Secondary Education Students of Callao}

\author{
Percy Madueño Ramos* \\ Universidad San Ignacio de Loyola, Lima, Perú \\ ORCID: https://orcid.org/0000-0003-3608-0770 \\ José Raúl Lévano Muchotrigo \\ Universidad Peruana de Ciencias Aplicadas, Lima, Perú \\ ORCID: https://orcid.org/0000-0002-2701-0693 \\ Aida Esther Salazar Bonilla \\ Universidad de San Martín de Porres, Lima, Perú \\ ORCID: https://orcid.org/0000-0002-2587-9782
}

Recibido 09-08-18 Revisado 10-11-18 Aprobado 07-05-19 En línea 22-07-19

\begin{tabular}{|c|c|}
\hline *Correspondencia & Citar como: \\
\hline Email:pmadueno@usil.edu.pe & $\begin{array}{l}\text { Madueño Ramos, P., Lévano Muchotrigo, R., \& Salazar } \\
\text { Bonilla, A. (2020). Conductas parentales y habilidades } \\
\text { sociales en estudiantes de educación secundaria del Callao. } \\
\text { Propósitos y Representaciones, 8(1), e234. doi: } \\
\text { http://dx.doi.org/10.20511/pyr2020.v8n1.234 }\end{array}$ \\
\hline
\end{tabular}




\section{Resumen}

El estudio tiene como propósito establecer la relación entre la percepción de las conductas parentales y las habilidades sociales en una muestra de 280 estudiantes, entre 11 y 14 años, de una Institución Educativa Estatal de la Provincia Constitucional del Callao. Se evidencia una relación directa entre la percepción de los adolescentes de las prácticas parentales positivas del padre y la madre con la presencia de habilidades sociales. El resultado del estudio se debe a la deseabilidad social, donde los adolescentes buscan transmitir una imagen culturalmente aceptable y evitar la crítica. Las implicancias de los resultados son discutidas en función de los objetivos planteados, de la realidad social y la realidad cultural de nuestro país.

Palabras clave: Sociedad educativa, Comportamiento de grupo; Habilidad; Adolescencia; Socialización.

\section{Summary}

The purpose of the study is to establish the relationship between the perception of parental behaviors and social skills in a sample of 280 students, between 11 and 14 years, of a State Educational Institution of the Constitutional Province of Callao. There is a direct relationship between the adolescents' perception of the positive parenting practices of the father and the mother with the presence of social skills. The result of the study is due to social desirability, where adolescents seek to transmit a culturally acceptable image and avoid criticism. The implications of the results are discussed in terms of the objectives set, the social reality and the cultural reality of our country.

Keywords: Educational Society, Group Behavior; Skill; Adolescence; Socialization.

\section{Introducción}

En el Perú, se aprecia un panorama donde niños, púberes que proceden de hogares disfuncionales (padres separados, maltratos psicológicos, maltrato físico y abandono), presentan conductas inadecuadas en sus comportamientos sociales, y esto se incrementa en familias de niveles socioeconómicos bajos.

Los estudios epidemiológicos de salud mental de niños y adolescentes de Lima y Callao del INSM (2016) informaron que 46,4\% de la población adolescente manifestó haber sufrido algún tipo de abuso en algún momento de su vida, siendo más prevalente el abuso psicológico 38,0\% y el abuso físico $25,8 \%$.

El Callao es una provincia con un alto índice de delincuencia, consumo de drogas, pandillaje, en ellas los niños y adolescentes son vulnerables ante estos problemas sociales y por ende al manejo de habilidades sociales que es objetivo del presente estudio.

Según Vargas (2017) los hijos expuestos a la violencia sufrida por sus madres pueden experimentar efectos negativos, independientes de la edad, que van desde problemas físicos hasta alteraciones emocionales, cognitivas y de la conducta.

La importancia de investigar la relación entre la percepción de las conductas parentales y las habilidades sociales en niños y adolescentes de nuestro medio, es conveniente porque nos enfrentamos con un panorama donde muchos niños que proceden de hogares disfuncionales (padres separados, maltrato psicológico y físico, abandono, etc.), presentan conductas inadecuadas en cuanto a sus comportamientos sociales, problema que merece mayor investigación ya que los padres son los mayores reforzadores, son fuentes de afecto y también los modelos de aprendizaje y comportamiento social; también la Institución educativa podría inculcar con mayor eficacia el 
dominio de habilidades sociales como parte de su rol formador personal de los estudiantes. Darling y Steinberg, en Merino, Díaz y Cohen (2003) manifiestan que:

"Uno de los estilos de crianza se caracteriza por una constelación de conductas parentales que crea un persistente clima interaccional en un amplio rango de contextos y situaciones" (p. 136).

Las conductas parentales están referidas al conjunto de comportamientos específicos de los padres para relacionarse con sus hijos y guiarlos al logro de metas de socialización (por ejemplo, brindarle halagos al niño con el propósito que aprenda nuevas tareas). Las conductas parentales tienen diversas funciones en el desarrollo infantil y del adolescente y en la dinámica familiar (Darling \& Steinberg, 1993).

Las conductas parentales están vinculadas con el desarrollo social, emocional y psicológico de los hijos. Las conductas que padres y madres emplean exclusiva y frecuentemente durante la interacción con sus hijos son denominadas conductas parentales (Ramírez, 2005). La conducta parental presenta características que se hayan en todas las sociedades humanas, Rohner (1975) halló dos características de la conducta parental: la aceptación y el rechazo. Según este autor, la aceptaciónrechazo parental consiste, en un extremo, se ubicarían los padres que muestran su amor y afecto hacia los hijos, y la disciplina es impuesta de forma inductiva y la comunicación entre padres e hijos es clara y abierta, basada en el respeto mutuo (Martínez \& Martínez, 2015) y en donde los hijos poseen confianza en sí mismos, adquieren responsabilidades, de modo que ellos mismo puedan solucionar sus problemas cotidianos. Esto permite que los niños y adolescentes desarrollen su autonomía y desarrollo personal, adquieran madurez en los aspectos psicosociales y sean menos propensos a la angustia y a externalizar sus problemas (Isaza \& Henao, 2012).

Sobre los tipos de conductas parentales, los investigadores Hazzard, Christiensen y Margolin (1983) definen dos tipos de conductas parentales percibidas por los niños y adolescentes dentro de sus entornos familiares, incluidos los patrones de crianza. Estos son: Percepciones positivas (Padres positivos y madres positivas) y Percepciones negativas (padre negativo vs madre negativa):

- Las percepciones positivas están dadas por: reforzamiento positivo, confort o comodidad, tiempo para conversar, participación en toma de decisiones, tiempo para estar juntos, evaluación positiva, aceptación de independencia, asistencia y afectividad no verbal.

- Las percepciones negativas están conformadas por: eliminación de privilegios, críticas, órdenes, castigo físico, gritos, amenazas, tiempo fuera, regaños e ignorar.

La información y la educación sobre los estilos parentales positivos y el establecimiento precoz de prácticas eficaces son importantes para la adaptación social y el éxito de un niño. En muchas situaciones, un estilo parental positivo: flexible, autoritativo y afectuoso es más beneficioso para el desarrollo social, cognitivo, moral y emocional del niño. No obstante, los estudios sobre interacciones padres e hijos deben ser actualizadas, para evaluar los resultados no solo en una diversidad de grupos culturales y socio-económicos, sino también en diferentes grupos etarios.

Tambien los estudios de Kershaw et al, (2014); manifiestan que es necesario examinar sus características y las variaciones que presentan en diversos contextos los niños y adolescentes, ya que las conductas parentales se han estudiado principalmente en las interacciones diádicas madre-hijo.

En las últimas décadas en las investigaciones psicológicas, el estudio de las habilidades sociales en los adolescentes ha obtenido un papel importante. Hoy en día se exige que las personas presenten desempeños sociales aceptables y más elaborados en los más variados contextos de 
interacción humana. Las habilidades sociales son consideradas importantes y fundamentales para los procesos de ajuste social de las personas (Angelino, 2014; Muñoz, Crespi \& Angrehs, 2011).

En suma, las habilidades sociales son capacidades o comportamientos específicos necesarios para relacionarnos e interactuar de forma efectiva y satisfactoria, desde actividades básicas hasta otras de características más avanzadas (Goldstein, 1989).

$\mathrm{Al}$ respecto, existe poca literatura o los estudios son escasos para poder hacer comparaciones o constatar similitudes con la presente investigación. La investigación tiene relevancia social y práctica porque posibilitará el diseño de programas preventivo - promocionales dirigidos a los padres de una institución educativa de la provincia del Callao para que tomen conciencia de que una parentalidad positiva crea las condiciones necesarias para que sus hijos puedan desarrollar sus capacidades y habilidades de la forma más completa posible. Asimismo, podrán adquirir mediante programas de entrenamiento el dominio de las habilidades sociales.

\section{Método}

La investigación corresponde a un diseño descriptivo correlacional porque examina e interpreta la relación o grado de asociación que existen entre las variables conductas parentales y habilidades sociales en alumnos de una I.E. de la provincia Constitucional del Callao. El propósito de los estudios correlacionales es describir la relación entre dos o más categorías, conceptos o variables en un momento determinado (Hernández, Fernández \& Baptista, 2014).

\section{Participantes}

La muestra con 280 participantes de ambos sexos, cuyas edades fluctúan entre los 11 y 14 años, pertenecientes a nivel socioeconómico bajo, los criterios para dicha categorización se han extraído de las fichas de matrícula de los estudiantes los cuales son: situación laboral precaria, inestable o desempleados, nivel educativo: analfabetos y/o con estudios básicos de primaria y en algunos casos inconclusos, los cuales estudian en una Institución Educativa Estatal del Callao. Provienen de familias disfuncionales, con carencias materiales, habitan viviendas de material prefabricado en su gran mayoría, los padres suelen dedicarse a trabajos eventuales y la zona donde habitan es considerada rural que no cuentan con servicios básicos de agua y desagüe.

\section{Criterios de inclusión}

Grado de instrucción: Alumnos de 5to, 6to grado de Primaria, $1^{\circ}$ y $2^{\circ}$ de secundaria, debido a que cuentan con un mejor nivel de comprensión de los instrumentos a utilizar.

Edad: Alumnos varones y mujeres de 11 a 14 años, debido a que dicho rango corresponde a los grados de estudios arriba mencionados.

Centro de estudios: Se consideró a la Institución Educativa de Gestión Estatal "César Vallejo" (Dulanto).

Sector poblacional: La presente investigación se realizó en la Provincia Constitucional del Callao donde se encuentra ubicado la Institución Educativa.

Nivel socioeconómico: Los alumnos pertenecen a los niveles socioeconómicos $\mathrm{C}$ y $\mathrm{D}$, en los que se agrupan el mayor porcentaje de familias (en ambos estratos: 69.5\%, APEIM, 2014).

Participación: La participación de los alumnos fue voluntaria. 


\section{Criterios de exclusión}

Disponibilidad para llenar los cuestionarios: si el alumno realizó conductas inadecuadas como bromear con sus compañeros, no prestar atención a las indicaciones brindadas por los evaluadores o realizó reiteradas preguntas por cada ítem a responder, su protocolo fue excluido.

Comprensión de los ítems: Los alumnos que presentaron dificultades para la comprensión de cada uno de los ítems, no se consideraron como parte de la muestra.

Nacionalidad extranjera: Se excluyó a los alumnos de procedencia extranjera.

\section{Instrumentos}

\section{Inventario de Percepción Parental (IPP)}

El Inventario de Percepción Parental (IPP) fue desarrollado por Hazzard, Christiensen, y Margolin (1983). Tiene por objetivo estudiar las percepciones de los niños de conductas parentales positivas y negativas de ambos progenitores. El instrumento consta de 18 ítems; 9 de ellos describen el comportamiento parental positivo (refuerzo positivo, la comodidad, de tiempo de conversación, la participación en la toma de decisiones, el tiempo juntos, la evaluación positiva, lo que permite independencia, la ayuda y el afecto no verbal) y 9 describen el comportamiento negativo de los padres (supresión del privilegio, la crítica, el mando, el castigo físico, gritos, amenazas, regaños, y haciendo caso omiso).

La primera adaptación en habla hispana, y específicamente en Perú fue la que realizaron Merino, Cohen y Diaz (2003), cuya traducción se efectuó por el método back-translation, el cual consiste en convertir un documento en ingles a otro idioma y posteriormente se traduce nuevamente al idioma original verificando así si existe alteración alguna. El presente instrumento que se utilizó en la investigación es apropiado para la exploración de las percepciones de los niños sobre las conductas parentales dentro de la crianza definidas por dos patrones comportamentales: conductas positivas y conductas negativas.

Los 18 ítems, presentan una lista de comportamientos de los padres respecto de sus hijos, donde el niño responderá si dicho comportamiento que se le presenta lo muestra su padre y madre ante él "nunca", "pocas veces", "algunas veces", "bastantes veces" o "muchas veces". Para ello, marca su respuesta haciendo un círculo o un aspa en una de las alternativas, sobre una escala de cinco puntos ( 0 a 4); las instrucciones y los ítems son leídos por la examinadora de los grupos evaluados. De esta prueba se obtienen cuatro Subescalas: Madre Positiva, Madre Negativa, Padre Positivo y Padre Negativo.

Los ítems para las subescalas Madre Positivo y Padre Positivo se enumeran en forma impar $(1,3,5,7,9,11,13,15,17)$ y miden los siguientes comportamientos: reforzamiento positivo, confortación, tiempo para conversar, participación en toma de decisiones, tiempo para estar juntos, evaluación positiva, a aceptación de independencia, asistencia y afectividad no verbal. Los ítems para las subescalas Madre Negativo y Padre Negativo son los números pares $(2,4,6,8,10,12,14$, $16,18)$ : los cuales evalúan conductas tales como remoción de privilegios, crítica, órdenes, castigo físico, gritos, amenaza, tiempo fuera, regaños, ignorar.

Respecto a los estudios de validez convergente, fue examinado por Hazzard en 1983 en una muestra de 75 niños de entre 5 y 13 años, utilizando medidas adicionales administradas a los niños y las medidas realizadas por sus padres. La escala mostró niveles aceptables de consistencia interna. No hay efectos de la edad de los niños; sin embargo, se encontraron los efectos de género del niño y 
de los padres. Los niños reportaron conductas parentales más positivas, especialmente para los padres, y ambos (tanto niños como niñas) reportaron más conductas negativas (en lo que respecta a la disciplina) de las madres.

La confiabilidad por consistencia interna (Cronbach, 1951) en el estudio original halló las siguientes estimaciones: Madre Positiva .84, Madre Negativa .87, Padre Positivo .88 y Padre Negativo .80. Considerando la edad, la consistencia en el rango 5 a 9 años la confiabilidad interna fue de .74 a .89 , y en el rango 10 a 13 años, de .81 a .87 .

En la investigación efectuada por Merino y Diaz (2003), en el Perú, respecto a la Confiabilidad de la prueba observaron que, las correlaciones ítem-test tuvieron un rango de .41 a .63 para Padre Positivo, .35 a .60 para Padre Negativo, .27 a .47 para Madre Positiva y .19 a .50 para Madre Negativa. Estas correlaciones tienden a ser, en general, similares a las encontradas por Hazzard et al. (1983) y, con dos excepciones, los ítems alcanzan óptimas correlaciones para los límites convencionalmente establecidos en el análisis de ítems. Los valores de alfa se mantienen en valores que van desde niveles tan bajos como .28 (segundo grado, Madre Positiva) hasta valores elevados como .86 (cuarto grado, Padre Positivo). Hay una tendencia a que los valores de alfa para las escalas referidas a la madre por lo común son bajos en relación con las escalas referidas al padre.

Los puntajes del Inventario de Percepción Parental - IPP, se relacionan previsiblemente a menor auto concepto y problemas de comportamiento (validez convergente) y por lo general no guardan relación con las medidas de desempeño del niño (validez discriminante).

Para el presente estudio, se obtuvo las características psicométricas mediante la evaluación de la estructura factorial y la consistencia interna. En el estudio realizado por Merino et al. (2003), las correlaciones inter - ítem fueron similares a los hallados por Hazzard et al. (1983).

\section{Lista de Chequeo de Habilidades Sociales de Goldstein.}

Cuyo autor es Arnold Goldstein en 1980, este instrumento fue traducido y adaptado a nuestro país por Ambrosio Tomás (1995). Tiene como objetivo identificar la presencia o déficit en las habilidades sociales en niños a partir de 12 años y adolescentes. Los objetivos de esta prueba son:

- Determinar las deficiencias y competencias que tiene una persona en sus habilidades sociales.

- Identificar el uso de la variedad de habilidades sociales, personales e interpersonales.

- Evaluar en qué tipo de situaciones las personas son competentes o deficientes en el empleo de una habilidad social.

La Lista de Chequeo de habilidades sociales consta de 50 preguntas, Las áreas o factores que mide son:

a) Grupo I: Primeras habilidades sociales: Escuchar, Iniciar una conversación, mantener una conversación, formular una pregunta, dar las gracias, presentarse, presentar a otras personas y hacer un cumplido.

b) Grupo II: Habilidades sociales avanzadas: Pedir ayuda, participar, dar instrucciones, seguir instrucciones, disculparse y convencer a los demás.

c) Grupo III: Habilidades para manejar sentimientos: Conocer los sentimientos propios, expresar los sentimientos propios, comprender los sentimientos de los demás, enfrentarse al enfado de otro, expresar afecto, resolver el miedo y Autorrecompensarse. 
d) Grupo IV: Habilidades alternativas a la agresión: Pedir permiso, compartir algo, ayudar a los otros, negociar, utilizar el autocontrol, defender los propios derechos, responder a las bromas, evitar los problemas con los demás y no entrar en peleas.

e) Grupo V: Habilidades para el manejo de estrés: Formular una queja, responder ante una queja, demostrar deportividad después de un juego, resolver la vergüenza, arreglárselas cuando es dejado de lado, defender a un amigo, responder a la persuasión, responder al fracaso, enfrentarse a mensajes contradictorios, responder a una acusación, prepararse para una conversación difícil y hacer frente a la presión de grupo.

f) Grupo VI: Habilidades de planificación: Tomar una decisión, discernir sobre la causa de un problema, establecer un objetivo, determinar las propias habilidades, recoger información, resolver problemas según la importancia, tomar la iniciativa y concentrarse en una tarea.

Dicha escala permite obtener la puntuación de cada uno de los factores y una puntuación total o global, lo que facilita diferenciar los perfiles de cada sujeto con puntuación total alta, que denotan aserción y habilidades sociales en distintos contextos, o de sujetos con puntuación global baja, que actúan de forma poco asertiva y con escasas competencias sociales (Salvador, De la Fuente \& Álvarez 2009).

La calificación es un procedimiento sencillo y directo que se ve facilitado por la estimación que hace el sujeto de su grado de competencia o deficiencia en que usa sus habilidades sociales, comprendidas en la lista de chequeo, el cual está indicando un valor cuantitativo. La puntuación máxima que obtener en un ítem es 5 y el valor mínimo es 1 . El puntaje total, que varía en función del número de ítems que responde el niño o adolescente en cada valor de 1 a 5 . Este puntaje como mínimo es 50 y como máximo 250 puntos. Mediante dichos puntajes, se trabajó con la media aritmética que es 150 .

Como Magnunson (1976), Guilford (1984) y Alarcón (1991); citados por Aguirre, (2002) señalan, la relación entre ítem y el test total puede expresarse como un coeficiente de correlación. Para la estandarización del presente instrumento se usó el coeficiente de Alfa de Cronbach cuya consistencia interna resultó con Alfa $=.98$ que significa confiabilidad alta y una Validez: $V$ de Aiken de .85

\section{Procedimiento}

Una vez obtenida la autorización de la Institución Educativa se procedió a coordinar con los docentes y tutores, los días y la hora adecuada para la aplicación de los instrumentos, de modo que faciliten el contacto con los alumnos. Se nos brindó las condiciones necesarias para la aplicación de los instrumentos (aula exenta de estímulos distractores y adecuada iluminación).

Los estudiantes fueron evaluados en su propio centro educativo, al cual asistían con regularidad durante el curso académico. Se explicó los objetivos de la investigación y el carácter confidencial de las respuestas.

Los instrumentos fueron aplicados en forma colectiva en cada aula; siendo el orden de los instrumentos: Ficha de datos sociodemográficos, Inventario de Percepción de Conductas Parentales y posteriormente la Escala de Habilidades Sociales. La duración aproximada fue de 40 minutos, teniendo en cuenta el correcto llenado de las hojas y monitoreando el desempeño de los alumnos. El proceso de aplicación de los instrumentos duró dos semanas (turno mañana y tarde). Cabe mencionar que se depuraron algunas pruebas por lo mencionado anteriormente. 
Es importante señalar que se obtuvo el consentimiento informado correspondiente de los padres de familia. Previamente se les hizo llegar un permiso para que pueda ser devuelto por el estudiante. Esta acción se coordinó directamente con la maestra de aula y la Dirección de la Institución Educativa.

Para el tratamiento estadístico de los datos, se utilizó el software SPSS versión 24 y se trabajó a dos niveles: con estadística descriptiva para el análisis de las variables a través de las medidas de tendencia central; mientras que para la contrastación de las hipótesis se trabajó con la estadística inferencial. Finalmente, el procesamiento de los datos fue realizado de manera automatizada usando el software estadístico aplicado SPSS, versión 24.0; presentando los resultados en tablas estadísticas de simple y doble entrada elaboradas según lo exigen las normas del Manual de Publicaciones de la American Psychological Association (APA).

\section{Análisis descriptivos y de normalidad}

Con respecto a la percepción de las conductas parentales positivas y negativas, se observa un mayor promedio en las prácticas positivas, padre positivo y madre positiva, aunque con un mayor promedio en madre positiva. Para ambos casos la tendencia en las puntuaciones ha ido hacia los puntajes altos en las prácticas positivas (asimetría negativa) y una tendencia hacia puntajes bajos en las prácticas negativas (asimetría positiva). En todos los casos la dispersión de los puntajes ha sido elevada (CV > 50\%; Pardo, Ruiz \& San Martín, 2015), lo que indica bastante alejamiento de las puntuaciones con respecto al promedio obtenido.

Con relación a las variables habilidades sociales y sus dimensiones, se aprecia un mayor promedio en las habilidades sociales avanzadas (HSA) y las habilidades de planificación (HP). Tanto en el puntaje total como en los puntajes de las seis dimensiones se observa una tendencia hacia puntuaciones altas (asimetría negativa) en las habilidades sociales por parte de los evaluados. Por último, la dispersión de los puntajes ha sido razonable $(\mathrm{CV}<50 \%)$, lo que indica que las puntuaciones han estado relativamente cercanas al puntaje promedio.

Tabla 1.

Estadísticos descriptivos de la percepción de conductas parentales positivas y negativas y las dimensiones de las HHSS

\begin{tabular}{lcccccccc}
\hline Dimensiones & $M / n^{\circ}$ items & Min. & Max. & $M$ & $D E$ & C.V & $g_{I}$ & $g_{2}$ \\
\hline Padre positivo & 2.18 & 0 & 32 & 17.45 & 8.54 & $48.94 \%$ & -0.29 & -0.89 \\
Padre negativo & 1.09 & 0 & 32 & 9.79 & 5.83 & $59.55 \%$ & 0.94 & 1.21 \\
Madre positiva & 2.68 & 1 & 32 & 21.47 & 7.30 & $34.00 \%$ & -0.57 & -0.24 \\
Madre negativa & 1.16 & 0 & 32 & 10.42 & 6.25 & $59.98 \%$ & 0.83 & 0.38 \\
HHSS & - & 78 & 223 & 166.98 & 27.10 & $16.23 \%$ & -0.35 & -0.34 \\
PHS & 3.51 & 10 & 40 & 28.10 & 5.79 & $20.60 \%$ & -0.19 & -0.42 \\
HSA & 3.69 & 8 & 25 & 18.47 & 3.91 & $21.17 \%$ & -0.18 & -0.70 \\
HPV & 3.28 & 7 & 35 & 22.97 & 5.52 & $24.03 \%$ & -0.22 & -0.27 \\
HA & 3.64 & 11 & 44 & 32.72 & 6.35 & $19.41 \%$ & -0.45 & 0.03 \\
HFE & 3.18 & 17 & 60 & 38.14 & 7.79 & $20.42 \%$ & -0.24 & -0.33 \\
HP & 3.32 & 10 & 38 & 26.58 & 5.27 & $19.83 \%$ & -0.35 & -0.22 \\
\hline
\end{tabular}


Nota $:$ HHSS $=$ habilidades sociales; PHS $=$ primeras habilidades sociales; HSA $=$ habilidades sociales avanzadas; $\mathrm{HPV}=$ habilidades para la vida; $\mathrm{HA}=$ habilidades alternativas; $\mathrm{HFE}=$ habilidades frente al estrés; HP = habilidades de planificación

Para analizar la normalidad se empleó la prueba de Kolmogorov-Smirnov, con el fin de contrastar la hipótesis nula $\left(\mathrm{H}_{0}\right)$ del que la distribución de los puntajes de las variables analizadas se aproxima a una distribución normal (Pardo \& San Martín, 2015); y, en base a ello, determinar la prueba estadística más apropiada para realizar los análisis inferenciales: prueba paramétrica o no paramétrica. Todos los resultados de dicho análisis se observan en la tabla 2.

Se realizó el análisis para la muestra y se encontró que los puntajes de las variables padre positivo y negativo y madre positiva y negativa y de las seis dimensiones de la variable de habilidades sociales (PHS, HSA, HPV, HA, HFE y HP) presentan una distribución que no se aproxima a una distribución normal $(p<.05)$. En ese sentido para calcular las correlaciones entre cada par de variables (correlaciones bivariadas) se aplicará el estadístico no paramétrico rho de Spearman.

Luego se realizó el análisis de normalidad de los puntajes de las variables tanto para el grupo de hombres como de mujeres. Se aprecia que, a excepción de las dimensiones PHS, HFE y HP en el grupo de las mujeres, todas las demás variables presentan distribuciones que no se aproximan a una distribución normal $(p<.05)$, por tal motivo para hacer comparaciones en los puntajes de cada una de las variables según el sexo de los participantes se aplicará la prueba no paramétrica $U$ de MannWhitney.

Finalmente, de acuerdo con los análisis de normalidad según la estructura familiar (monoparental, nuclear y extensa) para todas las variables se aplicó, la prueba H de Kruskal-Wallis.

Tabla 2.

Análisis de normalidad de la percepción de conductas parentales positivas y negativas y las dimensiones de las HHSS

\begin{tabular}{|c|c|c|c|c|c|c|}
\hline \multirow[b]{2}{*}{ Dimensiones } & \multirow[b]{2}{*}{$D$} & \multicolumn{2}{|c|}{ Sexo } & \multicolumn{3}{|c|}{ Estructura familiar } \\
\hline & & $\begin{array}{l}\text { Hombre } \\
(\mathrm{n}=135)\end{array}$ & $\begin{array}{c}\text { Mujer } \\
(\mathrm{n}=145)\end{array}$ & $\begin{array}{l}\text { Nuclear } \\
(\mathrm{n}=79)\end{array}$ & $\begin{array}{l}\text { Monoparental } \\
\quad(\mathrm{n}=72)\end{array}$ & $\begin{array}{c}\text { Extensa } \\
(\mathrm{n}=126)\end{array}$ \\
\hline Padre positivo & $0.09 * * *$ & $0.11 * * *$ & $0.10 * *$ & $0.11 *$ & $0.12 *$ & $0.10 * *$ \\
\hline Padre negativo & $0.10^{* * *}$ & $0.10 * *$ & $0.11^{* * *}$ & $0.13 * *$ & $0.16^{* * *}$ & $0.09 *$ \\
\hline Madre positiva & $0.06^{* *}$ & $0.09 *$ & $0.08^{*}$ & $0.11 *$ & $0.13 * *$ & $0.09 *$ \\
\hline Madre negativa & $0.11^{* * *}$ & $0.12^{* * *}$ & $0.12 * * *$ & $0.19 * * *$ & 0.09 & $0.11 * *$ \\
\hline PHS & $0.06^{* *}$ & 0.07 & $0.08^{*}$ & 0.09 & 0.10 & $0.08^{*}$ \\
\hline HSA & $0.08^{* * *}$ & $0.09 * *$ & $0.09 * *$ & $0.12 * *$ & 0.07 & $0.10 * *$ \\
\hline HPV & $0.08 * *$ & $0.09 *$ & $0.10 * *$ & 0.07 & 0.10 & $0.08 *$ \\
\hline HA & $0.07 * *$ & $0.09 *$ & $0.08 *$ & 0.08 & $0.10 *$ & 0.07 \\
\hline HFE & $0.07 * *$ & 0.07 & $0.09 * *$ & 0.09 & 0.07 & $0.08 *$ \\
\hline HP & $0.08 * * *$ & 0.07 & $0.10 * *$ & 0.12 & 0.08 & $0.10 * *$ \\
\hline
\end{tabular}

Nota: $D=$ estadístico de Kolmogorov-Smirnov; PHS = primeras habilidades sociales; HSA = habilidades sociales avanzadas; HPV = habilidades para la vida; HA = habilidades alternativas; HFE $=$ habilidades frente al estrés; $\mathrm{HP}=$ habilidades de planificación

$* * * p<.001$

$* * p<.01$

$* p<.05$ 


\section{Análisis correlacional}

En la tabla 3, se presentan los coeficientes de correlación de Spearman para cada par de variables analizadas. Se tomaron en cuenta para el análisis: (a) la dirección del coeficiente de correlación (positiva o negativa), (b) la significancia estadística y (c) el tamaño del coeficiente de correlación (Pardo \& San Martín, 2015).

Con respecto a la variable padre positivo se observa que presenta relaciones positivas y estadísticamente significativas con las seis dimensiones de las habilidades sociales, siendo la más fuerte la presentada con la dimensión habilidades sociales avanzadas (HSA; $r_{s}=.39, p<.001$ ) y la más débil con habilidades de planificación (HP; $\left.r_{s}=.19, p<.001\right)$; además presenta una relación negativa y significativa con la edad $\left(r_{s}=-.23, p<.001\right)$, lo cual significa que a mayor edad existe una menor percepción del niño de una práctica de crianza positiva por parte del padre. Así mismo, se aprecia con respecto a la variable padre negativo que no presentan ninguna relación estadísticamente significativa con las dimensiones de la variable habilidades sociales.

Por su parte, la variable madre positiva presenta también correlaciones positivas y estadísticamente significativas con las seis dimensiones de la variable habilidades sociales, siendo la más fuerte con habilidades sociales avanzadas (HSA; $r_{s}=.46, p<.001$ ) y la más débil con habilidades de planificación (HP; $r_{s}=.17, p<.001$ ); en líneas generales las correlaciones obtenidas han sido mayores en comparación a padre positivo, específicamente en primeras habilidades sociales (PHS), habilidades sociales avanzadas (HSA) y habilidades alternativas (HA). También presenta una relación negativa y significativa con la edad $\left(r_{s}=-.21, p<.001\right)$, lo cual significa que a mayor edad existe una menor percepción del niño de una práctica de crianza positiva por parte de la madre y también una mayor percepción de una práctica de crianza negativa materna $\left(r_{s}=.17, p<.001\right)$.

Finalmente, la variable madre negativa presenta una relación negativa y significativa con habilidades sociales avanzadas $\left(r_{s}=-.12, p<.05\right)$, aunque dicha relación presenta una magnitud baja. En síntesis, se observa una mayor asociación entre la percepción del niño de las conductas parentales positivas del padre y la madre con la presencia de habilidades sociales, dichas prácticas positivas tienen una mayor importancia en la presencia de las habilidades sociales avanzadas.

Tabla 3.

Análisis de correlaciones de la percepción de conductas parentales positivas y negativas y las dimensiones de las HHSS

\begin{tabular}{|c|c|c|c|c|c|c|c|c|c|c|c|}
\hline Variables & 1 & 2 & 3 & 4 & 5 & 6 & 7 & 8 & 9 & 10 & 11 \\
\hline 1. PP & - & & & & & & & & & & \\
\hline 2. $\mathrm{PN}$ & $-.21 * *$ & - & & & & & & & & & \\
\hline 3. MP & $.56^{* *}$ & $-.21 * *$ & - & & & & & & & & \\
\hline 4. $\mathrm{MN}$ & $-.24 * *$ & $.54 * *$ & $-.40 * *$ & - & & & & & & & \\
\hline 5. PHS & $.28 * *$ & .04 & $.34 * *$ & .03 & - & & & & & & \\
\hline 6. HSA & $.39 * *$ & -.07 & $.46^{* * *}$ & $-.12 *$ & $.58^{* *}$ & - & & & & & \\
\hline 7. HPV & $.24 * *$ & .01 & $.34 * *$ & -.02 & $.59^{* *}$ & $.61 * *$ & - & & & & \\
\hline 8. HA & $.21^{* *}$ & -.04 & $.36 * *$ & -.04 & $.52^{* *}$ & $.55^{* *}$ & $.54 * *$ & - & & & \\
\hline 9. HFE & $.21 * *$ & .05 & $.20 * *$ & .04 & $.51^{* *}$ & $.43^{* *}$ & $.50^{* *}$ & $.60 * *$ & - & & \\
\hline 10. HP & $.19 * *$ & -.04 & $.17 * *$ & .03 & $.48 * *$ & $.40^{* *}$ & $.51^{* *}$ & $.46^{* *}$ & $.60 * *$ & - & \\
\hline 11. Edad & $-.23 * *$ & .05 & $-.21 * *$ & $.17 * *$ & -.05 & -.07 & -.08 & -.01 & .08 & -.06 & - \\
\hline
\end{tabular}


Nota $:$ PP = padre positivo $; \mathrm{PN}=$ padre negativo $; \mathrm{MP}=$ madre positiva $; \mathrm{MN}=$ madre negativa; $\mathrm{PHS}$ $=$ primeras habilidades sociales; HSA $=$ habilidades sociales avanzadas; $\mathrm{HPV}=$ habilidades para la vida; $\mathrm{HA}=$ habilidades alternativas; $\mathrm{HFE}=$ habilidades frente al estrés; $\mathrm{HP}=$ habilidades de planificación.

$* * \mathrm{p}<.01$

$* \mathrm{p}<.05$

\section{Análisis comparativos}

Como análisis complementario se analizaron las diferencias de los puntajes obtenidos en las variables de estudio según algunas variables socio demográficas. A continuación, se presentan los resultados de comparar las prácticas parentales positivas y negativas y las dimensiones de las habilidades sociales según sexo (hombres y mujeres) y la estructura familiar (monoparental, nuclear y extensa).

\section{Diferencias según sexo}

En tabla 4 se presentan los resultados de las diferencias entre las dimensiones de las habilidades sociales según sexo. De acuerdo con los resultados se han encontrado diferencias estadísticamente significativas $(p<.05)$ en todas las dimensiones a excepción de las habilidades sociales avanzadas (HSA). En las cinco dimensiones donde se hallaron diferencias se aprecia que son las mujeres las que obtuvieron mayor puntaje promedio en comparación a los hombres, aunque al igual que en el caso anterior dichas diferencias encontradas son mínimas $(r<0.30)$.

Tabla 4.

Comparaciones de las dimensiones de Habilidades Sociales según sexo

\begin{tabular}{lcccccccc}
\hline & \multicolumn{2}{c}{ Mediana } & \multicolumn{2}{c}{ Media (DE) } & \multirow{2}{*}{$Z$} & $Z$ & $p$ & $R$ \\
\cline { 2 - 5 } & Hombre & Mujer & Hombre & Mujer & & & & \\
\hline PHS & 3.38 & 3.63 & $3.41(0.73)$ & $3.61(0.71)$ & 8144.50 & -2.43 & .015 & 0.15 \\
HSA & 3.60 & 3.80 & $3.61(0.80)$ & $3.77(0.76)$ & 8612.00 & -1.74 & .082 & 0.10 \\
HPV & 3.14 & 3.57 & $3.17(0.77)$ & $3.38(0.80)$ & 8307.50 & -2.19 & .029 & 0.13 \\
HA & 3.56 & 3.89 & $3.49(0.70)$ & $3.77(0.69)$ & 7543.50 & -3.32 & .001 & 0.20 \\
HFE & 3.17 & 3.33 & $3.08(0.61)$ & $3.27(0.67)$ & 7932.50 & -2.74 & .006 & 0.16 \\
HP & 3.25 & 3.50 & $3.20(0.66)$ & $3.44(0.64)$ & 7777.00 & -2.98 & .003 & 0.18 \\
\hline
\end{tabular}

Nota $:$ PHS $=$ primeras habilidades sociales; HSA $=$ habilidades sociales avanzadas; HPV= habilidades para la vida; $\mathrm{HA}=$ habilidades alternativas; $\mathrm{HFE}=$ habilidades frente al estrés; $\mathrm{HP}=$ habilidades de planificación; $d=$ tamaño del efecto

\section{Diferencias según estructura familiar}

En la tabla 5 se muestran las comparaciones entre la percepción de las prácticas de crianza positivas y negativas según la estructura familiar. En dicho análisis se observan diferencias estadísticas significativas solamente en la percepción de las prácticas de crianza positiva, tanto en padre positivo como en madre positiva según la estructura familiar. Al analizar específicamente entre qué grupos existían dichas diferencias, mediante la prueba post hoc de Scheffe, se encontraron tanto para padre positivo ( Dif $_{\text {monoparental-extensa }}=-0.49, p=.007, r=0.22$ ) como para Madre Positiva (Dif monoparental-extensa $=-0.40, p=.012, r=0.18$ ) que las diferencias encontradas estuvieron entre aquellos evaluados que pertenecen a una familia extensa en comparación a los que pertenecen a una familia monoparental. 
Para ambos casos los participantes que pertenecen a una familia extensa presentaron una mayor percepción de prácticas positivas de crianza de ambos padres en comparación a los que solo viven con uno de ellos, aunque las diferencias fueron pequeñas $(r<0.30)$.

Tabla 5.

Comparaciones de la percepción de las prácticas de crianza positivas y negativas según estructura familiar

\begin{tabular}{lccccc}
\hline & \multicolumn{5}{c}{ Media (DE) } \\
& Nuclear & Monoparental & Extensa & $\chi^{2}$ & $P$ \\
\hline Padre positivo & $2.25(0.99)$ & $1.86(1.07)$ & $2.34(1.07)$ & 10.11 & .006 \\
Padre negativo & $1.08(0.64)$ & $1.03(0.61)$ & $1.13(0.67)$ & 0.94 & .626 \\
Madre positiva & $2.65(0.91)$ & $2.46(0.99)$ & $2.85(0.82)$ & 7.14 & .028 \\
Madre negativa & $1.03(0.66)$ & $1.20(0.63)$ & $1.20(0.74)$ & 4.49 & .106 \\
\hline
\end{tabular}

Nota: $\chi^{2}=$ chi-cuadrado

En cuanto a las comparaciones de las dimensiones de las habilidades sociales según la estructura familiar de los evaluados, tal como se aprecia en la tabla 6, solamente se encontraron diferencias estadísticamente significativas $(\mathrm{p}<.05)$ en la dimensión habilidades sociales avanzadas (HSA). Al realizar la prueba post hoc de Schefee, se evidenció que específicamente las diferencias se dan entre los que pertenecen a una familia extensa en comparación a los que pertenecen a una familia monoparental ( Dif $_{\text {monoparental-extensa }}=-0.31, p=.029, r=0.18$ ), presentando una mayor puntuación los que pertenecen a una familia extensa; aunque la diferencias encontrada ha sido de magnitud pequeña $(r<0.30)$.

Tabla 6.

Comparaciones de las dimensiones de habilidades sociales según estructura familiar

\begin{tabular}{lccccc}
\hline & Media (DE) & & $\chi^{2}$ & $P$ \\
\hline PHS & $3.48(0.72)$ & $3.51(0.76)$ & $3.53(0.72)$ & 0.37 & .831 \\
HSA & $3.63(0.81)$ & $3.52(0.84)$ & $3.82(0.72)$ & 6.68 & .035 \\
HPV & $3.10(0.85)$ & $3.31(0.74)$ & $3.37(0.77)$ & 4.56 & .102 \\
HA & $3.55(0.73)$ & $3.59(0.76)$ & $3.71(0.66)$ & 2.13 & .345 \\
HFE & $3.12(0.70)$ & $3.14(0.65)$ & $3.22(0.61)$ & 1.52 & .468 \\
HP & $3.26(0.71)$ & $3.27(0.66)$ & $3.37(0.62)$ & 1.45 & .484 \\
\hline
\end{tabular}

Nota: PHS $=$ primeras habilidades sociales; HSA $=$ habilidades sociales avanzadas; HPV= habilidades para la vida; HA = habilidades alternativas; $\mathrm{HFE}=$ habilidades frente al estrés; $\mathrm{HP}=$ habilidades de planificación; $\chi^{2}=$ chi-cuadrado

Finalmente, podemos manifestar que los porcentajes de los tipos de familia a la cual pertenecen los adolescentes. Se ubica en primer lugar la familia extensa con un $45 \%$, seguido de la familia nuclear $28.2 \%$, en tercer y cuarto lugar las familias monoparentales, viven solo con la madre $23.2 \%$, viven solo con papá $2.5 \%$ y en última posición los que viven con otras personas o familiares $1.1 \%$.

\section{Discusión}

En este estudio se investigó la percepción de las conductas parentales en adolescentes, debido a la 
importancia que recae en los comportamientos de los padres con respecto a la forma de criar y/o educar a sus hijos. La conducta de los padres en relación con los hijos está íntimamente ligado a la imposición de normas, reglas y límites, pues desde los primeros años de vida, el bebé comienza a construir su referencial de actitudes por medio de la observación y del comportamiento del adulto. Recibe orientación al respecto de lo que es importante para preservar y defender la vida, como habilidades sociales y estímulos para crecer (Rinhel-Silva, Piemonte \& Rondini, 2012).

El tipo de apego y el modelo de relación construido por el niño con sus padres y otros miembros de la familia influyen en su competencia social con sus pares, así, el ambiente familiar puede ser tanto un contexto favorable como desfavorable en cuanto al desarrollo de los hijos, es decir, ser un factor de riesgo o protector para el normal desarrollo infantil (Risueño \& Cia, 2013).

Al analizar la relación entre la percepción de las conductas parentales y las habilidades sociales, se encontró una mayor asociación entre la percepción del niño de las prácticas parentales positivas del padre y la madre con la presencia de habilidades sociales avanzadas. Ello evidencia que el reforzamiento positivo, confortación, tiempo para conversar, participación en toma de decisiones, tiempo para estar juntos, evaluación positiva, aceptación de independencia, asistencia y afectividad no verbal, son cualidades que caracterizan a los padres y madres positivos; tal como corroboran las investigaciones del Perú e internacionales como la de Cárdenas (2013), Muñoz (2013), Santos (2012), Osorio, Rivas, Estévez, Calatrava y López de Burgo (2009), Bornstein y Bornstein (2007), que los hijos de padres con estilo de crianza positiva presentan mayores competencias sociales: interactúan adecuadamente, son empáticos, poseen autoconfianza, independientes y colaboradores (Del Prette \& Del Prette, 2012). Para Meir y Oros (2012), son muy significativos los vínculos y las relaciones que los adolescentes establecen con sus padres, ya que ejercen un impacto significativo a tal punto que se puede elaborar un perfil emocional característico de los hijos.

Sabbag (2010), acota que los jóvenes que perciben a sus padres con un mayor nivel de afecto y diálogo tienden a interactuar mejor con su entorno con un mayor nivel de independencia, lo cual indica que los padres positivos son responsivos afectivamente en la medida que se muestran cariñosos, brindan apoyo y proporcionan un ambiente estimulante y desafiante; además los padres que se comportan de manera adecuada en relación a las diversas situaciones vivenciadas en su papel parental y perciben los aspectos de la vida familiar y la comunicación como satisfactorios, transmiten a sus hijos un buen repertorio de habilidades sociales (Stasiak, Dobrianskyj \& Tucunduva, 2014). En este sentido, Risuenho y Cía. (2013), reiteran la relación existente entre un buen repertorio de habilidades sociales y las prácticas parentales positivas, en donde se aprecia que los padres saben establecer límites, corregir y supervisar, brindan un ambiente familiar protector a sus hijos y se desenvuelven asertivamente.

Asimismo, se aprecia que los padres con estilo parental positivo se caracterizan por ser menos autoritarios, más dispuestos a sugerir, insinuar y tratar de convencer sin imponerse ni dar órdenes (Van Barneveld, Rodríguez \& Robles, 2012). A decir de los propios padres, los aspectos de la crianza se conforman a partir de sus propios conocimientos, creencias y actitudes que están influenciados por el entorno social, la cultura dominante y la cultura heredada (Infante \& Martínez, 2016).

En la variable padre positivo se observa que las relaciones positivas son significativas con las seis dimensiones de las habilidades sociales, siendo la más fuerte las habilidades sociales avanzadas; por ello, Marin \& Piccinini (2009) destacan la importancia de la figura paterna; un padre no puede ser relegado a un segundo plano cuando se habla de desarrollo infantil y dinámica familiar.

En la variable padre negativo no presentan ninguna relación significativa con las dimensiones de la variable habilidades sociales. Así, Bornstein \& Bornstein (2007), detallaron que los estilos de crianza negativos a pesar de representar los extremos opuestos de las prácticas parentales no se relacionan con resultados positivos en las competencias sociales; los padres con un estilo de crianza negativo 
procuran modelar, controlar y evaluar el comportamiento del niño de acuerdo a un patrón rígido de comportamiento, que es considerado como patrón absoluto, también pueden caracterizarse por un bajo nivel de exigencia y escaso control, llegando a demostrar desinterés por sus hijos (Pereira, 2011). Algunos padres en su afán de monitorear a sus hijos acaban adoptando estrategias parentales que son percibidas como intrusivas y que afectan negativamente al adolescente, impidiendo su autonomía, autorregulación, manteniendo al joven emocionalmente dependiente de los padres o generando conductas de rebeldía hacia las reglas o normas propuestas por la familia (Bornstein \& Bornstein, 2007).

Desde esta perspectiva se podemos manifestar, que, en la correlación de variables, se puede verificar independientemente de ser práctica positiva o negativa que la mayoría de los adolescentes muestran comportamientos socialmente hábiles (Torres, 2016; Paes de Souza \& Löhr, 2015; Shimba \& De Castro, 2013; Leme et al., 2013; Santos, 2012; Doménique, 2012); esto indica que los adolescentes que participaron en dichas investigaciones tiene suficiente habilidad para expresar sus pensamientos, sentimientos, disconformidades hacia los demás, expresar su punto de vista, saber decir no, interrumpe una conversación que no desean seguir manteniendo, son producto de una decisión hecha por sí mismo de acuerdo a sus creencias, sentimientos, entre otros (Torres, 2016).

También en la variable madre positiva existe correlaciones positivas significativas con las seis dimensiones de la variable habilidades sociales, en general, las correlaciones obtenidas han sido mayores en comparación a padre positivo, específicamente en las primeras habilidades sociales (PHS), habilidades sociales avanzadas (HSA) y habilidades alternativas (HA). Marin y Piccinini (2009) resaltan que, independientemente del tipo de familia, muchas madres son más responsivas que el padre, establecen relaciones familiares que involucran amor, acercamiento y cooperación, lo que contribuye a que los hijos tengan más autonomía. Igualmente, Stasiak, Dobrianskyj y Tucunduva (2014) enfatizaron que las madres que tienen una comunicación asertiva, sus hijos son socialmente más hábiles, pues estas madres positivas conversan más con sus hijos, establecen límites, expresan sentimientos, incentivan, elogian y fortalecen las competencias de estos, además de servir como modelo adecuado para el desempeño social. De este modo, las conductas positivas maternas están más asociadas con la preocupación, cuidados y seguridad afectiva de los hijos.

Según Oliva y Arranz (2011), las hijas perciben estilos parentales más adecuados que los hijos; los hijos perciben a sus padres más rígidos, exigentes, con pautas educativas negativas, centradas en la irritabilidad, controladores con un mayor nivel de crítica y rechazo. Así mismo los adolescentes masculinos relatan que sus madres monitorean positivamente sus actividades más que a las hijas (Leme et al, 2013); estos resultados variados posiblemente se deban a que las familias tradicionales esperan que las hijas sean más sumisas a la autoridad, y para eso adoptaban estilos parentales controladores y a su vez los hijos tienen más libertad y autonomía (Leme et al, 2013); así mismo juegan un papel importante otros factores que pueden influir en la percepción de las prácticas parentales como: el estatus socioeconómico, la cultura de cada región o país.

Respecto a las conductas parentales maternas, Meir y Oros (2012), muestran indicadores que los adolescentes que perciben un alto control por parte de sus madres suelen sentirse menos tranquilos, con dificultad para sentir gratitud y menos satisfechos con la vida en comparación a aquellos que perciben un control moderado o bajo, y, la autonomía extrema otorgada por la madre influye significativamente sobre la experiencia de emociones positivas de los hijos. También Sabbag (2010), dice que el monitoreo adecuado que se caracteriza por la relación de diálogo e interacción positiva, ello es realizado a partir de la orientación y no a partir de conductas parentales intrusivas y hostiles, presentes en las madres negativas, los comportamientos con tales características pueden perjudicar la percepción de los hijos sobre sus padres y sobre sí mismos, además de perjudicar el desarrollo de su autonomía y sus competencias sociales. 
Por otro lado, es controversial los efectos de los diferentes estilos parentales sobre el desarrollo del niño en familias de bajo nivel socioeconómico, en situación de alto riesgo y que viven en barrios carentes servicios básicos. Al respecto Bornstein y Bornstein (2007) plantean que las prácticas parentales pueden hacer menos diferencia en las familias con bajo nivel socioeconómico, debido al mayor peso de los factores ambientales, tales como dificultades económicas y tasas de delincuencia más elevadas.

Así, Del Prette y Del Prette (2012) señalaron que tener un repertorio de habilidades sociales bien desarrolladas es imprescindible, pero no suficiente para el desempeño socialmente competente; uno de los factores que aumenta la posibilidad de alcanzar la competencia social es la capacidad que una persona tiene de monitorear su propio desempeño al relacionarse con otros.

Al analizar y comparar las dimensiones de las habilidades sociales según sexo y de acuerdo a los resultados se han encontrado diferencias significativas $(p<.05)$ en todas las dimensiones a excepción de las habilidades sociales avanzadas (HSA). En las cinco dimensiones se aprecia que las mujeres obtuvieron mayor puntaje promedio en comparación a los hombres. Existe evidencia en la literatura de que la adquisición y el desarrollo de las habilidades sociales están asociados a variables socio demográficas, entre ellas la variable sexo (Caballo, 2007; Fuentes, 2011; Isaza \& Henao, 2010; Lorente, 2014).

Torres (2016) encontró que ambos géneros presentaban similitud en sus habilidades sociales; los adolescentes de ambos sexos tienen competencias para expresar sus necesidades, afectos, preferencias o derechos sin experimentar ansiedad y respetando a los demás. A la par, el inicio de la adolescencia parece ser un momento en el que los contextos interpersonales se revisten de gran importancia; además, esta etapa es donde se da el establecimiento de las primeros relaciones afectivas y sexuales, así estos momentos son críticos para la expansión de los contactos sociales, lo que requiere el uso de más competencias para incrementar sus redes sociales, resolver problemas diarios y tomar decisiones con mayor autonomía (Campos, Del Prette \& Del Prette, 2014).

En tanto Lorente (2014) constató que los adolescentes varones muestran tendencia a mostrar menos preocupación ante las situaciones de otras personas, denotan una tendencia más agresiva y son proclives a presentar conductas antisociales; este autor afirma que los adolescentes quieren tener una identidad socialmente aceptable, por lo que en ocasiones tienden a mostrar conductas agresivas y evitan en la medida de sus posibilidades aquellos comportamientos que puedan considerarse femeninos.

Las diferencias halladas a favor de las mujeres podrían deberse a los cambios sociales producto de la globalización en los últimos años y la influencia de los estudios de género. Estos aportes en la actualidad, han producido grandes transformaciones en las formas de interactuar y de los vínculos mujer -hombre, permitiendo que la mujer se posicione y ocupe lugares de liderazgo ya no solo dentro de lo doméstico sino también en el mundo público (como jefa o puestos de línea de mando en las empresas); así vemos que un buen número de mujeres ocupan lugares destacados en la vida política, social y económica en la actualidad y esto puede servir como modelo para las mujeres adolescentes (Cohen, Caballero \& Mejail, 2012).

Otra respuesta a las variaciones en los resultados de las diversas investigaciones puede deberse a la metodología o los instrumentos de medida utilizados, y al mismo contexto; los estudiosos sugieren nuevos trabajos longitudinales, para verificar las diferencias de sexo en habilidades sociales y en diferentes momentos del desarrollo infantil (De Prette \& Del Prette, 2012). También Del Prette y del Prette (2009) han señalado que tener un repertorio de habilidades sociales bien desarrolladas es imprescindible, pero no suficiente para el desempeño socialmente competente; uno de los factores que aumenta la posibilidad de alcanzar la competencia social es la capacidad que una persona tiene 
de monitorear su propio desempeño al relacionarse con otros. Hay que recordar que los adolescentes valoran mucho las relaciones entre pares y tienden a formar su autoconcepto a través de su desempeño en el mundo social, siendo la aceptación por los demás un factor primordial. En comparación con la infancia, hay un aumento en la complejidad de las relaciones interpersonales en la adolescencia, pues en esta etapa se da más énfasis a la intimidad y la lealtad en las relaciones, lo que exige un mayor repertorio de habilidades sociales (Lorente, 2014).

En concordancia con el presente estudio Torres (2016) señala que los adolescentes procedentes de familias uniparentales, independientemente si viven con mamá o papá, tienen niveles promedios en sus habilidades sociales, les cuesta decir no y hacer peticiones, muestran incapacidad para iniciar o cortar una interacción, con el sexo opuesto suelen tener dificultad para empezar una conversación. Vallés (2008a) recalcaba la importancia de la asertividad, la cual definía como la capacidad de defender los propios derechos y expresar pensamientos, sentimientos y creencias de forma apropiada, sin transgredir los derechos de otras personas; pues esto posibilita el incremento de relaciones interpersonales saludables y duraderas. Leme et al. (2013) mencionan que las familias monoparentales y reconstituidas enfrentan desafíos constantes y patrones de interacción singulares que evidencian el impacto negativo sobre los hijos se debe a la falta de habilidades de comunicación, negociación y resolución de problemas entre las personas del mismo entorno familiar.

Finalmente, se analizan los resultados obtenidos al comparar la percepción de la práctica parental positiva y negativa según estructura familiar, se halló que los participantes que pertenecen a una familia extensa presentaron una mayor percepción de prácticas positivas de crianza de ambos padres en comparación a los que solo viven con uno de ellos, aunque las diferencias fueron mínimas; en este contexto, es importante estudiar el impacto de la diversidad familiar sobre el desarrollo y competencia de los adolescentes, porque cada vez es más frecuente en la sociedad occidental, la inserción de los adolescentes en una configuración familiar diferente a la formada por padres e hijo de la primera unión conyugal.

Los estudios señalan implicancias negativas de las configuraciones parentales, en particular de las madres solteras, porque asumen con mayor dificultad los roles parentales y mayores niveles de comportamiento parental negativo debido al uso de prácticas de crianza ineficaces y a un menor involucramiento, menor interacción, escaso control y supervisión de los hijos, aunado a la falta de apoyo y soporte social y condición económica; esto hace que el niño responda con un comportamiento social inadecuado y presente más conductas negativas que los hijos de otras composiciones familiares (Marin \& Piccinini, 2009).

Con relación a los diferentes contextos familiares, constitución, organización, estructura y la interacción entre los miembros, se aprecia que estos interfieren en los problemas de comportamiento, en las conductas de los niños y en la calidad de relacionarse con los hermanos, percibiéndose que cuando la pareja tiene una buena relación, existe una mejor calidad en la interacción entre los hijos (Stasiak et al., 2014; Isaza y Henao, 2012; Sabbag, 2010). En estos trabajos se encontraron más problemas de conducta en familias de madres solteras o familias reconstituidas en comparación a las familias nucleares. Sin embargo y en contraste Capano y Ubach (2013); Marin y Piccinini (2009); Paes de Sousa y Löhr (2015) no encontraron diferencias significativas en cuanto al estilo de crianza de los padres de diferentes configuraciones familiares; incluso afirman que los niños pueden desarrollarse sin prejuicios en hogares de madres solteras, especialmente en relación a sus características socio demográficas, psicológicas y sociales; la ausencia del padre puede ser compensada por la dedicación de las madres u otros factores, como el apoyo social recibido.

Un resultado positivo, del presente estudio, se debe a la deseabilidad social, termino que corresponde a la propensión para transmitir una imagen culturalmente aceptable y de acuerdo con las normas sociales, buscando la aprobación y evitando la crítica en situaciones de evaluación, 
Domínguez, Aguilera, Acosta, Navarro y Ruiz (2012) acotan que la deseabilidad social es una variable genuina en sí misma y representa una necesidad auténtica para agradar a los demás. Por tanto, las personas con una elevada deseabilidad social tendrán mayor tendencia a dar respuestas que consideran serán bien aceptadas por el entorno independientemente de ser o no, negando la asociación personal las opiniones o comportamientos socialmente desaprobados.

\section{Referencias}

Bornstein, B., \& Bornstein, M. (2007). Estilos de prácticas parentales y desarrollo social del niño. EE.UU: University of Pennsylvania; National Institute of Child Health and Human Development.

Caballo, V. (2007). Manual de Evaluación y Entrenamiento de las habilidades sociales (4a Ed.). España: Siglo Veintiuno Editores S.A.

Campos, J., Del Prette, A., \& Del Prette, Z. (2014). Depresión en la adolescencia: habilidades sociales y variables socio demográficas como factores de riesgo / protección. Estudios y pesquisas en Psicología, 14(2), 408-428. doi: https://doi.org/10.12957/epp.2014.12645

Cárdenas, K. (2013). Asociación entre estilos de socialización parental y habilidades sociales del adolescente en una Institución Educativa Nacional - VMT (Tesis de licenciatura). Escuela de Enfermería Padre Luis Tezza, Lima.

Capano, A., \& Ubach, A. (2013). Estilos Parentales, parentalidad positiva y formación de padres. Ciencias Psicológicas, 7(1), 83-95. doi: https://doi.org/10.22235/cp.v7i1.41

Cohen, S., Caballero, V., \& Mejail, S. (2012). Habilidades sociales, aislamiento y comportamiento antisocial en adolescentes en contextos de pobreza. Acta Colombiana de Psicología, 15(1), $11-20$. http://editorial.ucatolica.edu.co/ojsucatolica/revistas_ucatolica/index.php/acta-colombianapsicologia/article/view/192

Del Prette, Z., \& Del Prette, A. (2012). Social skills and behavior analysis: Historical proximity and new issues. Perspectivas en análisis del comportamiento, $1(2), 104-115$. doi: https://doi.org/10.18761/perspectivas.v1i2.35

Del Prette, Z., \& Del Prette, A. (2009). Adolescencia y factores de riesgo: La importancia de las habilidades sociales educativas.

Doménique, Y. (2012). Actitudes maternas y habilidades sociales en niños de 5 años en la Red $N^{\circ}$ 10 del Callao (Tesis de maestría). Universidad San Ignacio de Loyola, Lima.

Domínguez, A., Aguilera, S., Acosta, T., Navarro, G. \& Ruiz, Z. (2012). La deseabilidad social revalorada: Más que una distorsión, una necesidad de aprobación social. Acta de Investigación Psicológica, 2(3), 808-825. Recuperado de https://pdfs.semanticscholar.org/ab3c/a28babc5de82dff53550d6290b2081e847bc.pdf

Fuentes, K. (2011). Habilidades sociales y convivencia escolar (Tesis de Licenciatura). Universidad Academia de Humanismo Cristiano, Lima.

Hernández, R., Fernández, C., \& Baptista, P. (2014). Metodología de la Investigación. México: McGraw-Hill.

Infante, A., \& Martínez, J. (2016). Concepciones sobre la crianza: el pensamiento de madres y padres de familia. Liberabit, 22(1), 31-41. doi: https://doi.org/10.24265/liberabit.2016.v22n1.03

Instituto Nacional de Salud Mental Honorio Delgado (2016). Maltrato infantil. Recuperado de http://www.insm.gob.pe/

Isaza, L., \& Henao, G. (2010). El desempeño en habilidades sociales en niños, de dos y tres años de edad, y su relación con los estilos de interacción parental. Electronic Journal of Research in Educational Psychology, 8(22), 1051-1076. Recuperado de http://ojs.ual.es/ojs/index.php/EJREP/article/view/1453

Isaza, L., \& Henao, G. (2012). Influencia del clima socio familiar y estilos de interacción parental sobre el desarrollo de habilidades sociales en niños y niñas. Persona, 15, 253-271. 
Kershaw, T., Murphy, A., Lewis, J., Divney, A., Albritton, T., Magriples, U., \& Gordon, D. (2014). Family and relationship influences on parenting behaviors of young parents. Journal of Adolescent Health, 54(2), 197-203. doi: http://dx.doi.org/10.1016/j.jadohealth.2013.08.012

Leme, V., Del Prette, Z., \& Coimbra, S. (2013). Prácticas educativas parentales y habilidades sociales de adolescentes de diferentes configuraciones familiares. Revista Psico, 44(4), 560-570. Recuperado de http://revistaseletronicas.pucrs.br/ojs/index.php/revistapsico/article/view/12559/0

Lorente, S. (2014). Efecto de la competencia social, la empatía y la conducta prosocial en adolescentes (Tesis doctorado). Universitat de Valencia, Valencia. Recuperado de http://hdl.handle.net/10550/36209

Marín, A., \& Piccinini, C. (2009). Familias uniparentales: la madre soltera en la literatura. Revista Psico, 40(4), 422-429. Recuperado de http://revistaseletronicas.pucrs.br/revistapsico/ojs/index.php/revistapsico/article/view/2683

Meir, L., \& Oros, L. (2012). Percepción de las prácticas parentales y experiencia de emociones positivas en adolescentes. Revista de Psicología, 8(16), 73-84. Recuperado de https://ri.conicet.gov.ar/handle/11336/19108

Muñoz, C., Crespi, P. \& Angrehs, R. (2013). Habilidades Sociales. Madrid: Paraninfo S.A.

Oliva, A. \& Arranz, E. (2011). Nuevas familias y bienestar infantil. Sevilla: Universidad de Sevilla.

Osorio, A., Rivas, S., Estévez, J., Calatrava, M. \& López de Burgo, C. (2009). Evaluación de los estilos educativos parentales en una muestra de estudiantes filipinos: implicaciones educativas. Revista Panamericana de Pedagogía, 14, 13-37. Recuperado de http://dadun.unav.edu/handle/10171/19106

Paes de Souza, H., \& Löhr, T. (2015). Relación entre prácticas parentales y habilidades sociales de niños de enseñanza básica de una escuela pública. PsicolArgum, 33(80), 255-269. Recuperado de http://www2.pucpr.br/reol/pb/index.php/pa?dd1=16144\&dd99=view\&dd98=pb

Pardo, A. \& San Martín, R. (2015). Análisis de datos en ciencias sociales y de la salud II (2 $2^{\mathrm{a}}$. ed.). Madrid: Síntesis.

Pardo, A., Ruiz, M. A. \& San Martín, R. (2015). Análisis de datos en ciencias sociales y de la salud $I$ ( $2^{\mathrm{a}}$. ed.). Madrid: Síntesis.

Pereira, R. (2011). Adolescentes en el siglo XXI. Entre impotencia, resiliencia y poder. España: Morata.

Ramírez, M. A. (2005). Padres y desarrollo de los hijos: prácticas de crianza. Estudios Pedagógicos, 31(2), 167-177. doi: http://dx.doi.org/10.4067/S0718-07052005000200011

Rinhel-Silva, C., Piemonte, E. \& Rondini, C. (2012). Familia, adolescencia y estilos parentales. Estudios de Psicología, 29(2), 221-230. Recuperado https://psycnet.apa.org/record/201222555-008

Risuenho, S., \& Cia, F. (2013). Pais e filos: habilidades educativas parentais e habilidades sociais infantiles. VIII Encontro da Associação brasileira de pesquisadores em educação especial. Recuperado

de http://www.uel.br/eventos/congressomultidisciplinar/pages/arquivos/anais/2013/AT092013/AT09-004.pdf

Sabbag, G. (2010). Análisis de las prácticas parentales y de las habilidades sociales educativas maternas en la interacción con los hijos adolescentes (Tesis de Maestría). Universidad Estatal Paulista, São Paulo.

Santos, L. (2012). El clima social familiar y las habilidades sociales de los alumnos de una institución educativa del Callao (Tesis de maestría). Universidad San Ignacio de Loyola, Lima.

Shimba, R., \& De Castro, M. (2013). Un estudio de la relación entre estilos parentales y habilidades sociales. Revista Augustus, 18(36), 57-68. Recuperado de http://apl.unisuam.edu.br/revistas/index.php/revistaaugustus/article/view/1981-

1896.2013v18n36p57 
Stasiak, G., Dobrianskyj, L., \& Tucunduva, C. (2014). Calidad en la interacción familiar y estrés parental y su relación con el autoconcepto, habilidades sociales y problemas de comportamiento en los hijos. Revista Psico, 45(4), 494-501. doi: http://dx.doi.org/10.15448/1980-8623.2014.4.15846

Torres, V. (2016). Estilos de socialización parental y habilidades sociales en estudiantes del nivel secundario de una I.E.P. de Lima Metropolitana. (Tesis de licenciatura). Universidad Peruana Unión, Lima.

Vallés, A. (2008a). Habilidades sociales e inteligencia emocional para el afrontamiento del estrés.

Van Barneveld, H., Rodríguez, B. \& Robles, E. (2012). La percepción de la crianza en padres, madres e hijos adolescentes pertenecientes al mismo núcleo familiar. Liberabit, 18(1), 75-82. Recuperado de http://ojs3.revistaliberabit.com/publicaciones/revistas/RLE_18_1_lapercepcion-de-la-crianza-en-padres-madres-e-hijos-adolescentes-pertenecientes-al-mismonucleo-familiar.pdf

Vargas, H. (2017). Violencia contra la mujer infligida por su pareja y su relación con la salud mental de los hijos adolescentes. Revista Médica Herediana, 28(1), 48-58. doi: https://dx.doi.org/10.20453/rmh.v28i1.3074 